\title{
Stochastics and thermodynamics for equilibrium measures of holomorphic endomorphisms on complex projective spaces
}

\author{
Michał Szostakiewicz • Mariusz Urbański • \\ Anna Zdunik
}

Received: 10 February 2013 / Accepted: 10 January 2014 / Published online: 14 February 2014 C The Author(s) 2014. This article is published with open access at Springerlink.com

\begin{abstract}
It was proved by Urbański and Zdunik (Fund Math 220:23-69, 2013) that for every holomorphic endomorphism $f: \mathbb{P}^{k} \rightarrow \mathbb{P}^{k}$ of a complex projective space $\mathbb{P}^{k}, k \geq 1$, there exists a positive number $\kappa_{f}>0$ such that if $J$ is the Julia set of $f$ (i.e. the support of the maximal entropy measure) and $\phi: J \rightarrow \mathbb{R}$ is a Hölder continuous function with $\sup (\phi)-\inf (\phi)<\kappa_{f}$ (pressure gap), then $\phi$ admits a unique equilibrium state $\mu_{\phi}$ on $J$. In this paper we prove that the dynamical system $\left(f, \mu_{\phi}\right)$ enjoys exponential decay of correlations of Hölder continuous observables as well as the Central Limit Theorem and the Law of Iterated Logarithm for the class of these variables that, in addition, satisfy a natural co-boundary condition. We also show that the topological pressure function $t \mapsto P(t \phi)$ is real-analytic throughout the open set of all parameters $t$ for which the potentials $t \phi$ have pressure gaps.
\end{abstract}

Communicated by S. G. Dani.

The research of M. Urbański supported in part by the NSF Grant DMS 1001874. The research of M. Szostakiewicz and A. Zdunik supported in part by the Polish NCN Grant N N201 607940.

\section{Szostakiewicz}

Institute of Mathematics of the Polish Academy of Sciences, Interdisciplinary Centre for Mathematical and Computational Modelling, Warsaw University, ul. Pawińskiego 5a, Block D, 5th Floor,

02-106 Warsaw, Poland

e-mail: M.Szostakiewicz@icm.edu.pl

M. Urbański

Department of Mathematics, University of North Texas, Denton, TX 76203-1430, USA

e-mail: urbanski@unt.edu

URL: http://www.math.unt.edu/ urbanski

\section{A. Zdunik (凶)}

Institute of Mathematics, Warsaw University, ul. Banacha 2, 02-097 Warsaw, Poland

e-mail: A.Zdunik@mimuw.edu.pl 
Keywords Holomorphic dynamics - Complex projective spaces - Julia sets · Topological pressure - Equilibrium states · Hölder continuous potentials · PerronFrobenius operators · Exponential decay of correlations · Central Limit Theorem · The Law of Iterated Logarithm

Mathematics Subject Classification (1991) Primary 37D35; Secondary 32H50 . $37 \mathrm{C} 40 \cdot 28 \mathrm{D} 05$

\section{Introduction}

Fix an integer $k \geq 1$. Let $f: \mathbb{P}^{k} \rightarrow \mathbb{P}^{k}$ be a holomorphic endomorphism of degree $d \geq 2$ of the complex projective space $\mathbb{P}^{k}$. Denote by $J=J(f)$ the Julia set of the map $f: \mathbb{P}^{k} \rightarrow \mathbb{P}^{k}$, i.e. the topological support of the measure of maximal entropy. The map $f: \mathbb{P}^{k} \rightarrow \mathbb{P}^{k}$ is called regular if its exceptional set $E=E(f)$ does not intersect the Julia set $J=J(f)$. Recall that the exceptional set $E=E(f)$ is a proper algebraic, totally invariant subset contained in the critical set, such that, given $a \in \mathbb{P}^{k}$, the sequence of the measures $d^{-k n}\left(f^{n}\right)^{*} \delta_{a}$ equally distributed on the preimages of the point $a$ converges to the measure of maximal entropy if and only if $a \notin E$. For $k=1$ the set $E(f)$ is either empty, or of cardinality $2\left(z \mapsto z^{ \pm d}\right)$, or of cardinality 1 (polynomials). Obviously, for $k=1$ the set $E(f)$ never intersects $J(f)$, so in dimension 1 , every map is regular. In dimension $k>1$, we put this as an additional assumption, although we do not know any example of a holomorphic map $f: \mathbb{P}^{k} \rightarrow \mathbb{P}^{k}$ for which the intersection $E(f) \cap J(f)$ would be nonempty. Moreover, it is known that the set $E(f)$ is empty for a generic holomorphic map $f: \mathbb{P}^{k} \rightarrow \mathbb{P}^{k}$ (see [3, Lemma 1.5.6]).

Let $\phi: J(f) \rightarrow \mathbb{R}$ be a continuous function, in the sequel frequently referred to as a potential. By $P(\phi)$ we denote the (classical) topological pressure of the potential $\phi$ with respect to the dynamical system $f: J(f) \rightarrow J(f)$. Its definition and a systematic account of properties can be found for example in [11]. If $\mu$ is a Borel probability $f$-invariant measure on $J(f)$, we denote by $\mathrm{h}_{\mu}(f)$ its Kolmogorov-Sinai metric entropy. The relation between pressure and entropy is given by the following celebrated Variational Principle.

$$
\mathrm{P}(\varphi)=\sup \left\{\mathrm{h}_{\mu}(f)+\int \varphi d \mu\right\},
$$

where the supremum is taken over all Borel probability $f$-invariant measures $\mu$, or equivalently, over all Borel probability $f$-invariant ergodic measures $\mu$. The measures $\mu$ for which

$$
\mathrm{h}_{\mu}(f)+\int \phi d \mu=\mathrm{P}(\phi)
$$

are called equilibrium states for the potential $\phi$. The main theorem proved in [15] is this. 
Theorem 1.1 Let $f$ be a regular holomorphic endomorphism of a complex projective space $\mathbb{P}^{k}, k \geq 1$, of algebraic degree $d$. Then there exists a positive number $\kappa_{f}$, where $\log d \geq \kappa_{f}>0$, such that if $\phi: J(f) \rightarrow \mathbb{R}$ is a Hölder continuous function with $\sup (\phi)-\inf (\phi)<\kappa_{f}$ (we then say that $\phi$ has a pressure gap), then $\phi$ admits a unique (hence ergodic) equilibrium state $\mu_{\phi}$ on J. This equilibrium state is equivalent to a fixed point of the normalized dual Perron-Frobenius operator. In addition the dynamical system $\left(f, \mu_{\phi}\right)$ is $K$-mixing. In the case when the Julia set $J$ does not intersect any periodic irreducible algebraic variety contained in the critical set of $f$, we have that $\kappa_{f}=\log d$.

Remark 1.2 We would like to remark that in [2] the key assumption (pressure gap) was that

$$
\sup (\phi)<\mathrm{P}(\phi),
$$

and this assumption was then repeated in [12]. It corresponds to the assumption

$$
(k-1) \log d+\sup (\phi)<\mathrm{P}(\phi)
$$

in the multidimensional ( $k$ is the dimension) case; it reduces to (1.2) if $k=1$. In the present paper the weakest assumption is that $\sup (\phi)-\inf (\phi)<\log d$, which is somewhat stronger than (1.3). In fact we inherited it from [15], where it permitted us to estimate almost painlessly from below the eigenvalues of the Perron-Frobenius operator. With the assumption (1.3) it would be an additional hard technical issue to, the already technically heavy paper [15].

The main object of study in our paper will be the dynamical system $\left(f, \mu_{\phi}\right)$. We shall show in Theorem 7.6 that this system enjoys exponential decay of correlations of Hölder continuous observables as well as the Central Limit Theorem and the Law of Iterated Logarithm for the class of these variables that, in addition, satisfy a natural co-boundary condition. We also show in Theorem 6.1 that the topological pressure function $t \mapsto P(t \phi)$ is real-analytic throughout the open set of all parameters $t$ for which the potentials $t \phi$ have pressure gaps.

This paper is self-contained in the sense that all notions used are introduced and all the steps leading to the main theorems are explained. Many proofs, however, are close to those for the 1-dimensional case dealt with in [12], and pointing out the particular fragment of [12] we refer the reader to this paper for some proofs.

Another class of invariant measures was considered by Dupont in [5]. These are images (under certain natural coding) of Gibbs measures for some Hölder continuous potentials, on an appropriate symbolic space. Stochastic properties were proved for these measures in [5] (see also [4]). However, the approach used in that paper cannot be applied for our class of the most natural invariant measures. In our case, the potential is a regular (Hölder continuous) function defined directly in the dynamical space $\mathbb{P}^{k}$. But its composition with the coding map is (usually) highly irregular.

\section{Good holomorphic inverse branches}

Assumptions. Throughout the paper, we keep the following two assumptions: 
(1) $f: \mathbb{P}^{k} \rightarrow \mathbb{P}^{k}$ is a regular holomorphic map.

(2) $\phi: J(f) \rightarrow J(f)$ is a Hölder continuous potential satisfying $\sup \phi-\inf \phi<\kappa_{f}$.

Under these assumptions, the main theorem from [15] holds (see Theorem 1.1 above). First, we recall that in [15] the equilibrium measure was constructed as a fixed point of the corresponding Perron-Frobenius operator. More precisely, assuming (which is always possible) that $P(\phi)=0$, we finally obtain the invariant density $\rho_{\phi}$ as a unique fixed point of the operator $\mathcal{L}_{\phi}: C(J(f)) \rightarrow C(J(f))$ :

$$
\mathcal{L}_{\phi}(\rho)(x)=\sum_{y \in f^{-1}(x)} \exp \phi(y) \rho(y),
$$

where the inverse images of critical values of $f$ are counted with multiplicities. As usually, we shall denote by $S_{n} \phi$ the sum

$$
S_{n} \phi(y)=\phi(y)+\cdots+\phi\left(f^{n-1}(y)\right) .
$$

The unique conformal measure $m_{\phi}$ is obtained as a fixed point of the dual operator $\mathcal{L}_{\phi}^{*}$. The measure $\mu_{\phi}$ is then equal to

$$
d \mu_{\phi}=\rho_{\phi} d m_{\phi}
$$

Note that $\exp (-\phi)$ is the Jacobian of the measure $m_{\phi}$ while

$$
\exp (-\tilde{\phi})=\frac{\rho_{\phi} \circ f}{\rho_{\phi}} \exp (-\phi)
$$

is the Jacobian of the measure $\mu_{\phi}$, where

$$
\tilde{\phi}=\log \rho_{\phi}-\log \rho_{\phi} \circ f+\phi .
$$

Note also that for the normalized Perron-Frobenius operator $\mathcal{L}_{\tilde{\phi}}$, defined analogously to $\mathcal{L}_{\phi}$, with $\phi$ replaced by $\tilde{\phi}$, we have

$$
\mathcal{L}_{\tilde{\phi}}(\mathbb{1})=\mathbb{1}
$$

From now on, we assume that $P(\phi)=0$.

Given an open connected subset $W$ of $\mathbb{P}^{k}$ and given an integer $n \geq 1$, we denote by $I_{n}(W)$ the collection of all connected components of $f^{-n}(W)$. If $V \in I_{n}(W)$ and $\left.f\right|_{V} ^{n}: V \rightarrow W$ is a bijection (equivalently an injection), we set

$$
f_{V}^{-n}:=\left(\left.f\right|_{V} ^{n}\right)^{-1}: W \rightarrow V
$$

We denote the collection of all such components by $\mathrm{PG}_{n}(W)$ and refer to them as pre-good components. Of course, if $V \in \mathrm{PG}_{n}(W)$, then the map $f_{V}^{-n}: W \rightarrow V$ 
is a holomorphic homeomorphism from $W$ to $V$. We call it the holomorphic inverse branch of $f^{n}$ from $W$ to $V$.

It follows from our assumptions that there exists $\delta>0$ such that

$$
\inf (\phi) \geq \sup (\phi)-(1-\delta) \log d
$$

Using the above notation, we have

$$
\begin{aligned}
1=\int \mathcal{L}_{\phi} \mathbb{1} d m_{\phi} & =\int \sum_{w \in f^{-1}(z)} \exp \phi(w) d m_{\phi} \geq d^{k} \exp (\inf \phi) \\
& =\exp (k \log d+\inf (\phi)) \\
& \geq \exp ((k-1+\delta) \log d+\sup \phi)) .
\end{aligned}
$$

Using this, we get

$$
\exp (\phi(z)) \leq \exp (-(k-1+\delta) \log d)=d^{-(k-1+\delta)} .
$$

Thus,

$$
\exp \left(S_{n} \phi(z)\right) \leq d^{-n(k-1+\delta)}
$$

and

$$
\exp \left(S_{n} \tilde{\phi}(z)\right) \leq M d^{-n(k-1+\delta)}
$$

where $M=\sup \left(\rho_{\phi}\right) / \inf \left(\rho_{\phi}\right)$. The proof of the following proposition is now immediate.

Proposition 2.1 If $V \in P G_{n}(W)$, i.e. if $V$ is a pre-good component of $f^{-n} W$, then

$$
\mu_{\phi}(V) \leq M d^{-(k-1+\delta)} \mu_{\phi}(W) .
$$

Given $l \in \mathbb{N}$, denote by $P C_{l}$ the postcritical set of the map $f^{l}$, i.e.

$$
P C_{l}=f^{l}\left(\operatorname{Crit}\left(f^{l}\right)\right) \text {. }
$$

Our main technical tool in this section is the following.

Lemma 2.2 Fix $\delta$ as above and some $0<\delta^{\prime}<\delta$. Let $\theta=d^{-\frac{\delta^{\prime}}{2}}<1$. Then there exists $\eta>0$ such that, for every $\varepsilon>0$ there exist $l \in \mathbb{N}$ and $C<\infty$ such that if $B$ is an arbitrary open ball centered at a point from the Julia set $J$, and if $B$ is disjoint from the set $P C_{l}$ then, with $B^{\prime \prime}=\eta B$, for every integer $n \geq l$ there exists a subset of components of $G_{n}\left(B^{\prime \prime}\right) \subset P G_{n}\left(B^{\prime \prime}\right)$ with the following properties.

(a) If $V \in G_{n+1}\left(B^{\prime \prime}\right)$, then $f(V) \in G_{n}\left(B^{\prime \prime}\right)$.

(b) If $V \in G_{n}\left(B^{\prime \prime}\right)$, then $\operatorname{diam}(V) \leq C \theta^{n}$.

(c) $\mu_{\phi}\left(\bigcup G_{n}\left(B^{\prime \prime}\right)\right) \geq(1-\varepsilon) \mu_{\phi}(B)$, where $\bigcup G_{n}\left(B^{\prime \prime}\right):=\bigcup\left\{V: V \in G_{n}\left(B^{\prime \prime}\right)\right\}$ 
(d) There exists $\beta>0$ such that

$$
\mu_{\phi}\left(\bigcup B_{n}\left(B^{\prime \prime}\right)\right) \leq \exp (-\beta n)
$$

where $B_{n}\left(B^{\prime \prime}\right)$ consists of all connected components of the sets $f^{-1}(V), V \in$ $G_{n-1}\left(B^{\prime \prime}\right)$ that do not belong to $G_{n}\left(B^{\prime \prime}\right)$ and $\bigcup B_{n}\left(B^{\prime \prime}\right):=\bigcup\left\{V: V \in B_{n}\left(B^{\prime \prime}\right)\right\}$.

(e) $\sup _{z} B_{n}(z) \leq \exp (-\beta n)$, where $B_{n}(z)=\sum_{w \in f^{-n}(z) \cap \bigcup B_{n}\left(B^{\prime \prime}\right)} \exp S_{n} \tilde{\phi}(w)$.

Proof It can be deduced from the proof of the similar result for the measure of maximal entropy, see [6, p.1599]. The difference is that, here, we have to estimate not only the number of "bad" components $B_{n}$, which is sufficient for the case of maximal measure, but also the value of the measure $\mu_{\phi}$ of their union. The estimate (d) can be easily deduced from [6], using Proposition 2.1 above (the estimate of the measure of a pregood component), as we will sketch briefly below. Of course (d) follows from (e), and (c) follows from (d) for $l$ large enough.

We put some details below.

In the first step-exactly in the same way as Guedj-we show that, the family $f^{-1}\left(P G_{n}\left(\rho_{n-1} B\right) \backslash P G_{n}\left(\rho_{n} B\right)\right)$ (meaning: all components in $f^{-1} P G_{n}\left(\rho_{n-1} B\right)$, which are not components in $P G_{n}\left(\rho_{n} B\right)$ and $\left.\rho_{n}=\sum_{l}^{n} j^{-2}\right)$ consists of at most $D n^{4(k-1)} d^{(k-1) n}$ components, where $D$ is a constant depending on the dimension $k$ and on the degree of the map $f$, but independent of $n$. In the light of Proposition 2.1 this gives estimates on the number and measure of components in $P G_{n}(\rho B)$ where $\rho=\lim \rho_{n}=\sum_{l}^{\infty} j^{-2}$.

In the second step we define $G_{n}\left(B^{\prime \prime}\right), B^{\prime \prime}=a \rho B$, to be:

$$
G_{n}\left(B^{\prime \prime}\right):=\left\{V \in P G_{n}\left(B^{\prime \prime}\right): \operatorname{diam}(V) \leq C \theta^{n}=d^{-\delta^{\prime} / 2}\right\}
$$

where $a$ and $C$ are constants which will be specified below.

If we prove that $P G_{n}\left(B^{\prime \prime}\right) \backslash G_{n}\left(B^{\prime \prime}\right)$ consists of at most $d^{\left(k-1+\delta^{\prime}\right) n}$ components, then we will be able to estimate the function $B_{n}(z)$ by $M d^{-\left(\delta-\delta^{\prime}\right) n}\left(D n^{4(k-1)}+1\right)$. The latter is smaller than $\exp (-\beta n)$ (with $\beta$ equal, say $\left(\delta-\delta^{\prime}\right) / 2$ ) for all $n \geq l$ if $l$ has been chosen large enough.

To show that, first—again, in the same way as Guedj-we notice that

$$
\sum_{V \in P G_{n}\left(B^{\prime}\right)} \int_{\mathbb{P}^{k-1}} \operatorname{Area}\left(f_{V}^{-n}\left(\Delta_{\gamma}\right)\right) d \nu(\gamma) \leq d^{(k-1) n},
$$

where $v$ denotes Fubini-Study measure on $\mathbb{P}^{k-1}, \Delta_{\gamma}:=L_{\gamma} \cap \rho B$ and $L_{\gamma}$ is the complex line passing through the center of $B$ in the direction $\gamma$. Thus, there are at most $d^{\left(k-1+\delta^{\prime}\right) n}$ components $V \in P G_{n}(\rho B)$, for which the inequality

$$
\int \operatorname{Area}\left(f_{V}^{-n}\left(\Delta_{\gamma}\right)\right) d v(\gamma) \leq d^{-\delta^{\prime} n}
$$


does not hold. If on some $V=f_{V}^{-n}(\rho B)$ it does hold, then the diameter of $f_{V}^{-n}(1 / 4 \rho B)$ is small. To prove it, we shall use the same argument as in [6], which we include for the convenience of the reader. Consider $V \in P G_{n}(\rho B)$ on which (2.3) does hold. Then on a set $A \in \mathbb{P}^{k-1}$ of measure bigger than $1 / 2$ we have $\operatorname{Area}\left(f_{V}^{-n} \Delta_{\gamma}\right) \leq 2 d^{-\delta^{\prime} n}$. Now, an argument using extremal length (see lemma in Appendix of [1]) we get that $\operatorname{diam}\left(f_{V}^{-n} 1 / 2 \Delta_{\gamma}\right) \leq 2 c d^{-\delta^{\prime} n / 2}$ for $\gamma \in A$ where $c$ is a constant depending on the dimension $k$ only. Then, a theorem in [14] (see [3], Theorem 1.4.10 for a convenient formulation) gives us that $\operatorname{diam}\left(f_{V}^{-n} a \rho B\right) \leq 2 c d^{-\delta^{\prime} n / 2}$, where, again $a$ is a constant depending only on the dimension of the space but independent of $f$ and of $n$. This gives the statement of the Lemma with $\eta=a \rho$.

For $\eta$ as in the statement of Lemma 2.2, put $B^{\prime}=\frac{1}{2} \eta B=\frac{1}{2} B^{\prime \prime}$. Applying Cauchy's formulas for partial derivatives, we directly obtain from Lemma 2.2 the following.

Corollary 2.3 With the hypotheses of Lemma 2.2 we have that there exists a constant $C_{\eta}>0$ such that

$$
\left\|D f_{V}^{-n}(z)\right\| \leq C_{\eta} \theta^{n}
$$

for all $n \geq 0$, all $V \in G_{n}\left(B^{\prime \prime}\right)$ and all $z \in B^{\prime}=\frac{1}{2} \eta B$.

Fixing $l$. We now keep the setting of Lemma 2.2 with $\varepsilon:=1 / 2$. This gives the value of iterate $l$, which we fix at this step of the construction.

Notation. From now on, we frequently write $G_{n}, B_{n}$, instead of $G_{n}\left(B^{\prime}\right), B_{n}\left(B^{\prime}\right)$. As in Lemma 2.2, item (e), we denote

$$
B_{n}(z)=\sum_{w \in f^{-n}(z) \cap \bigcup B_{n}\left(B^{\prime}\right)} \exp S_{n} \tilde{\phi}(w) .
$$

Since we will need to pass several times to an iterate, we now introduce the following.

Notation. $G_{n}^{f^{m}}$ denotes the family of good components $G_{m n}$. Next, $B_{n}^{f^{m}}$ equals, roughly speaking $f^{-m}\left(G_{n-1}^{f^{m}}\right) \backslash G_{n}^{f^{m}}$, or, more precisely, $B_{n}^{f^{m}}$ consists of those components of the sets $f^{-m} V$ (where $V \in G_{n-1}^{f^{m}}$ ), which are not in $G_{n}^{f^{m}}$. Accordingly, we put

$$
B_{n}^{f^{m}}(z)=\sum_{w \in f^{-n m}(z) \cap \bigcup B_{n}^{f^{m}}\left(B^{\prime}\right)} \exp S_{n m} \tilde{\varphi}(w) .
$$

Lemma 2.4 There exists a constant $C=C(\beta)$ such that, for all $q \geq l, n \geq 2$, and all $z \in B^{\prime}$, we have that

$$
B_{n}^{f^{q}}(z) \leq C \exp (-\beta(n-1) q) .
$$

Proof First, notice that

$$
B_{n}^{f^{q}}=f^{-q}\left(\bigcup G_{q(n-1)}\right) \backslash \bigcup G_{q n}=\bigcup_{(n-1) q<k \leq n q} f^{-(n q-k)}\left(\bigcup B_{k}\right) .
$$


Thus, remembering that $\mathcal{L}_{\tilde{\phi}}(\mathbb{1})=\mathbb{1}$, and, consequently, for every set $A$, and every $m, \mathcal{L}_{\tilde{\phi}}\left(\mathbb{1}_{f^{-m}(A)}\right)=\mathbb{1}_{A}$ !, we get that

$$
\begin{aligned}
B_{n}^{f^{q}}(z) & =\mathcal{L}_{\tilde{\phi}}^{n q} \mathbb{1}_{B_{n}^{f q}}(z)=\sum_{(n-1) q<k \leq n q} \mathcal{L}_{\tilde{\phi}}^{n q} \mathbb{1}_{f^{-(n q-k)}\left(B_{k}\right)}(z)=\sum_{(n-1) q<k \leq n q} \mathcal{L}_{\tilde{\phi}}^{k} \mathbb{1}_{B_{k}}(z) \\
& =\sum_{(n-1) q<k \leq n q} B_{k}(z) \leq \sum_{(n-1) q<k \leq n q} \exp (-\beta k) \\
& \leq \frac{\exp (-\beta)}{1-\exp (-\beta)} \exp (-\beta(n-1) q) .
\end{aligned}
$$

We are done.

Corollary 2.5 Let $q \geq l$; put $g=f^{q}$. There exists a constant $C_{q}$ such that, for all $n \geq 1$ and all $z \in B^{\prime}$,

$$
B_{n}^{g}(z):=B_{n}^{f^{q}}(z) \leq C_{q} \exp (-\beta n q)
$$

Proof This directly follows from Lemma 2.4, by putting

$$
C_{q}:=\exp (\beta q) \max \left\{C(\beta), \sup _{z} B_{1}^{g}(z)\right\} \text {. }
$$

In what follows, we shall need to pass to yet another iterate $h=g^{r}$. The corollary below gives an explicit bound for $B_{n}^{h}(z)$.

Corollary 2.6 Let, as before, $g=f^{q}, q \geq l$. Now, let $r \geq 1$ and $n \geq 2$. Then

$$
B_{n}^{h}(z):=B_{n}^{f^{q r}}(z) \leq C_{q} \exp \left(\frac{-n \beta q r}{2}\right)
$$

where $C_{q}$ is another constant depending on $q$, but independent of $r$ and $n$.

Proof Writing

$$
\bigcup B_{n}^{h}=\bigcup_{(n-1) r<k \leq n r} g^{-(n r-k)}\left(\bigcup B_{k}^{g}\right)
$$

and using the same reasoning as in the proof of Lemma 2.4, we get

$$
\begin{aligned}
B_{n}^{h}(z) & \leq \sum_{(n-1) r<k \leq n r} B_{k}^{g}(z) \leq \sum_{(n-1) r<k \leq n r} C_{q} \exp (-\beta k q) \\
& \leq C_{q}(1-\exp (-\beta q))^{-1} \exp (-\beta(n-1) q r) .
\end{aligned}
$$

Since $n \geq 2$,we have $n-1 \geq n / 2$, and the result follows. 
We fix here the constant $C_{q}$, for which both Corollaries 2.5 and 2.6 hold.

The most significant consequence for a component to belong to $G_{n}$ is this.

Corollary 2.7 There exists a constant $C_{l}$ such that, for all $n$, for every good component $V$ of the set $f^{-n}\left(B^{\prime}\right)$, i.e. $V \in G_{n}$, and for all $x, y \in V$, we have that

$$
\frac{\exp \left(S_{n} \phi(x)\right)}{\exp \left(S_{n} \phi(y)\right)} \leq C_{l} \text { and } \frac{\exp \left(S_{n} \tilde{\phi}(x)\right)}{\exp \left(S_{n} \tilde{\phi}(y)\right)} \leq C_{l}
$$

Proof This follows directly from the expanding property along good branches (see item (b) in Lemma 2.2) and Hölder continuity of $\phi$.

\section{Selection of the root ball}

First, note that the topological support of the measure $\mu_{\phi}$ is equal to $J(f)$. This means that every open ball intersecting $J(f)$ has a positive measure $\mu_{\phi}$.

The proof of the following lemma is rather standard. A slightly different version can be found in [12, Lemma 9].

Lemma 3.1 Let $a<b$ be two real numbers. If $\mu$ is a Borel finite measure on $[a, b]$, then for every $\lambda>1$ large enough there exists a point $c \in(a, b)$, in fact a measurable set of positive Lebesgue measure of such points $c$, such that

$$
\mu\left(\left(c-\lambda^{-n}, c+\lambda^{-n}\right)\right) \leq \tilde{\lambda}^{-n}
$$

for all integers $n \geq 1$, where $\lambda=\tilde{\lambda}^{3}$.

As a straightforward consequence of the abstract Lemma 3.1, we shall prove the following.

Lemma 3.2 For every point $w \in J \backslash P C_{l}$ there exists a ball $B$ centered at $w$ such that $\frac{2}{\eta} B \cap P C_{l}=\emptyset$ and

$$
\mu_{\phi}\left(B\left(\partial B, \lambda^{-n}\right)\right) \leq \tilde{\lambda}^{-n} .
$$

with $\lambda, \tilde{\lambda}$ taken from Lemma 3.1, and $\eta$, taken from Lemma 2.2.

Proof Take any $R>0$ so small that $\frac{2}{\eta} B(w, R) \cap \mathrm{PC}_{l}=\emptyset$ and consider the map $P: \bar{B}(w, R) \rightarrow[0, R]$ given by the formula $P(z)=\|z-w\|$. Applying Lemma 3.1 to the measure $\mu_{\phi} \circ P^{-1}$ the assertion of Lemma 3.2 immediately follows.

\section{Fine inducing scheme}

In this section, starting from the ball selected above, we define good and very good pullbacks defined on $B$. By pullbacks we mean the holomorphic branches of $f^{-q r}$ (where $r$ will be precisely determined below). Using these selected "very good" pullbacks we shall define, in Sect. 5, the induced map (an infinite Iterated Function System). 
We assume that an integer $r \geq 1$ is so large that

$$
C_{\eta} \theta^{r} \leq 1 / 4
$$

where the constant $C_{\eta}$ comes from Corollary 2.3. More requirements on $r$ will be imposed later. Also, $q$ will be fixed later on. Having $q$ and $r$ chosen, we put, as before:

$$
h=f^{q r}: \mathbb{P}^{k} \rightarrow \mathbb{P}^{k}
$$

and, to simplify the notation,

$$
\begin{aligned}
\phi_{0} & :=\sum_{j=0}^{q r-1} \phi \circ f^{j}, \\
\tilde{\phi}_{0} & :=\sum_{j=0}^{q r-1} \tilde{\phi} \circ f^{j}=\sum_{j=0}^{q r-1} \phi \circ f^{j}-\log \rho_{\phi} \circ f^{q r-1}+\log \rho_{\phi} .
\end{aligned}
$$

Denote by $\mathcal{L}_{0}$ the normalized Perron-Frobenius operator associated to the map $h$ : $J \rightarrow J$. It is given by the formula

$$
\mathcal{L}_{0}(\zeta)(x)=\sum_{y \in h^{-1}(x)} \zeta(y) \exp \left(\tilde{\phi}_{0}\right)(y)=\frac{1}{\rho_{\phi}(x)} \mathcal{L}_{\phi}^{q r}\left(\zeta \cdot \rho_{\phi}\right)(x)
$$

Note that $\mathcal{L}_{0}(\mathbb{1})=\mathbb{1}$. Again, this is important in the forthcoming proofs.

Remark 4.1 Actually, since $\rho_{\phi}$ is a continuous function, $\mathcal{L}_{0}$ acts (as well as $\mathcal{L}_{\phi}$ ) as a continuous linear operator in the space $C(J)$. However, we do not use the continuity of $\rho_{\phi}$. The only fact we need is that $\rho_{\phi}$ is bounded away from 0 and $\infty$.

The following, quite general Lemma was proved in [12].

Lemma 4.2 Assume that $Q \subset \mathbb{P}^{k}$ is a set for which there exists $\gamma>0$ such that

$$
\mathcal{L}_{0}\left(\mathbb{1}_{Q}\right)(x)>\gamma
$$

for every $x \in J(f)$. Then there exist $\alpha \in(0,1)$, an integer $n_{0} \geq 0$, and $\delta>0$, all three depending on $\gamma$ only (in particular independent of $r \geq 1$ ), such that for all $n \geq n_{0}$ we have that,

$$
\mu_{\phi}\left(\left\{x \in J(f): \#\left\{0 \leq i \leq n: h^{i}(x) \in Q\right\} \leq \alpha n\right\}\right)<\exp (-\delta n) .
$$

Remark 4.3 In order to make this lemma useful for us, we need some preparation. Note that, given $h$ (recall that $h=f^{r q}$ ) and an open set $Q$, it is easy to find $\gamma$ for which (4.2) is satisfied (the existence of such $\gamma$ follows directly from the topological exactness of the map). However, at this point we need to show that, for our particular 
choice of $Q=Q_{r}$ (see Lemma 4.4 below), $\gamma$ can be chosen to be independent of $r$, as $r$ will be modified in the course of the proof.

Fixing $q$. We choose $q$ at this point; it will not be modified in the rest of the proof. On the contrary, $r$ has not been fixed yet, and some additional conditions on $r$ will appear later on. Recall that $l$ has been already chosen in Lemma 2.2, applied for $\varepsilon=\frac{1}{2}$. The ball $B$, and, consequently $B^{\prime}=(\eta / 2) B$, has been fixed in Lemma 3.2. Since $f_{\mid J(f)}$ is topologically exact, there exists $N$ such that $f^{N}\left(B^{\prime}\right) \supset J(f)$. Finally, we put $q=\max (N, l)$.

Lemma 4.4 For every integer $q \geq l$ there exists $\gamma>0$ such that for every integer $r \geq 1$, we have for every $z \in J(f)$ that,

$$
\mathcal{L}_{0}\left(\mathbb{1}_{Q}\right)(z) \geq \gamma
$$

where

$$
Q:=Q_{r}:=\bigcup\left\{V: V \in G_{(r-1) q}\left(B^{\prime}\right)\right\} .
$$

Proof As a straightforward consequence of Lemma 2.2, particularly its item (c), and (2.4), we get the following.

$$
\mathcal{L}_{\tilde{\phi}}^{q(r-1)} \mathbb{1}_{Q}(z) \geq\left(2 C_{l}\right)^{-1}
$$

for all $z \in B$. By the choice of $q$, we have $f^{q}(B) \supset J(f)$. Thus, for every $x \in J$ there then exists $y \in B$ such that $f^{q}(y)=x$. Applying (4.4) we can then write

$$
\begin{aligned}
\mathcal{L}_{0}\left(\mathbb{1}_{Q}\right)(x) & \geq \exp \left(S_{q} \tilde{\phi}(y)\right) \mathcal{L}_{\tilde{\phi}}^{q(r-1)} \mathbb{1}_{Q}(y) \\
& \geq\left(2 C_{l}\right)^{-1} \exp \left(S_{q} \tilde{\phi}(y)\right) \\
& \geq\left(2 C_{l}\right)^{-1} \exp \left(\inf \left(S_{q} \tilde{\phi}\right)\right)
\end{aligned}
$$

Setting $\gamma$ to be the last number of this formula finishes the proof. It is important to notice here that $\gamma$ depends on $q$, but it is independent of $r$.

Definition 4.5 We say that a point $z \in B^{\prime}$ has a good pullback of length $n \geq 1$ if $h^{n}(z) \in B^{\prime}$ and $V_{n}(z)$, the connected component of $h^{-n}\left(B^{\prime}\right)$ containing $z$, belongs to $G_{n}^{h}\left(B^{\prime}\right)$. We then frequently refer to this component just as a (good) pullback.

Definition 4.6 We further say that such a good pullback $V_{n}(z)$ is very good if for every $0 \leq j \leq n-1$, there exists $z_{j} \in h^{n-j}\left(V_{n}(z)\right)$ such that

$$
\operatorname{dist}\left(z_{j}, \partial B^{\prime}\right)>\lambda^{-j}
$$

Remark 4.7 Note that if $r \geq 1$ is chosen so large that,

$$
C_{\eta} \theta^{r q}<\lambda^{-1}
$$


(see Corollary 2.3 and also (4.1)), and $V_{n}$ is a very good pullback then the sets $h^{n-j}\left(V_{n}\right)$ do not intersect $\partial B^{\prime}$; in particular $V_{n}$ is entirely contained in $B^{\prime}$.

Having fixed $q$, we find $\gamma$, according to the statement of Lemma 4.4, and $\alpha$, given by Lemma 4.2, applied for $Q=Q_{r}$. Again, it is important to note that one can find a common value $\gamma$ (and thus also $\alpha$ ), valid for all $r$.

The proof of Lemma 4.8 below is similar to Lemma 17 from [12]. As in Lemma 4.2 let $Z_{n}$ be the set of all points $x \in B^{\prime}$ for which

$$
\#\left\{0<i \leq n: h^{i}(x) \in B^{\prime} \text { and } g^{i r-(r-1)}(x) \in Q\right\}>\alpha n
$$

It follows from Lemma 4.2 that $\mu_{\phi}\left(Z_{n}\right) \geq \mu\left(B^{\prime}\right)-\exp (-\delta n)$.

\section{Lemma 4.8 Let}

$$
Y_{n}:=\left\{x \in Z_{n}: \#\left\{0 \leq j \leq n: \text { the pullback } V_{j}(x) \text { is } \operatorname{good}\right\}<(\alpha / 2) n\right\} .
$$

Then

$$
\mu_{\phi}\left(Y_{n}\right) \leq\left(3 C_{q}\right)^{n} \exp (-r q n \beta \alpha / 4),
$$

where the constants $\beta, C_{q}$ come from Corollary 2.6.

Thus, with $r \geq 1$ sufficiently large (depending on $q$ and the constants above) we have that

$$
\mu_{\phi}\left(Y_{n}\right)<\exp (-r q n \beta \alpha / 8)
$$

Proof As we have mentioned, the proof is in the spirit of the proof of Lemma 17 from [12]. Nevertheless, it requires several modifications, so we outline it below and refer to [12] for omitted details. Recall that $g$ and $h$ have been chosen so that $h=g^{r}$ and $g^{r-1}(Q)=B^{\prime}$.

Let $x \in Y_{n}$, and consider a part of the trajectory of $x$ :

$$
x, g(x), \ldots, g^{r}(x)=h(x), \ldots, g^{(n-1) r}(x)=h^{n-1}(x), g^{(n-1) r+1}(x)
$$

We now describe the 'configuration' $M$ of integers, defined by the trajectory of $x$. First, the set of indexes $I=\{1, \ldots, n\}$ is divided into two disjoint groups:

$I_{1}=\left\{j \in I\right.$ : such that $g^{r j-(r-1)}(x) \in Q$ (in particular, $h^{j}(x)=g^{r j}(x)=$ $\left.g^{r-1}\left(g^{r j-(r-1)}(x)\right) \in B\right)$, but the pullback $V_{j}(x)$ is not good $\}$

$I_{2}=\left\{j \in I\right.$ : such that either $g^{r j-(r-1)}(x) \in Q$ and the pullback $V_{j}(x)$ is good or $\left.g^{r j-(r-1)}(x) \notin Q\right\}$

Note that there are at most $n-\alpha n / 2$ elements in $I_{2}$, since $x$ visits $Q$ at least $\alpha n$ times, but there are less than $\alpha n / 2$ good pullbacks. The configuration $M=M(x)$ is a sequence $m_{1}>m_{1}^{\prime} \geq m_{2}>m_{2}^{\prime} \geq \cdots \geq m_{k}>m_{k}^{\prime}$ defined as follows: First, let

$$
m_{1}=\max \left\{m \in I_{1}\right\}
$$


Since the pullback $V_{m_{1}}(x)$ is not good, there exists $m_{1}^{\prime}<m_{1}$ such that $h^{m_{1}^{\prime}}(x) \in$ $B_{m_{1}-m_{1}^{\prime}}$. If $m_{1}^{\prime}-m_{1}=1$, then we can say more: then $h^{m_{1}^{\prime}}(x) \in B_{r}^{g}$, as $g\left(h^{m_{1}^{\prime}}(x)\right)=$ $g^{r m_{1}-(r-1)}(x) \in Q=G_{r-1}^{g}$.

Next, $m_{2}$ is chosen as the largest element $\left(\leq m_{1}^{\prime}\right)$, belonging to the group $I_{1}$. Inductively, we find $m_{2}^{\prime}, m_{3}, m_{3}^{\prime}$, etc, until $m_{k}^{\prime}$ such that all $1, \ldots, m_{k}^{\prime}$ are in $I_{2}$.

In this way, the set $I=\{1, \ldots, n\}$ has been divided into 'blocks' $\left(m_{i}^{\prime}, m_{i}\right]$ and 'gaps'. Since every element in a gap is in $I_{2}$, the sum of lengths of blocks $\Delta m_{i}:=m_{i}-$ $\left.m_{i}^{\prime}\right)$ is at least $\alpha n / 2$.

Consider the set $Y_{n}^{M}$ of points in $Y_{n}$, sharing the same configuration $M$. The set $Y_{n}^{M}$ is contained in $C:=f^{-m_{1}^{\prime}}\left(W_{\triangle m_{1}}\right) \cap f^{-m_{2}^{\prime}}\left(W_{\triangle m_{2}}\right) \cap \ldots f^{-m_{k}^{\prime}}\left(W_{\triangle m_{k}}\right)$, where $W_{m}$ is equal to $B_{m}$ for $m \geq 2$, and $W_{1}=B_{r}^{g}$. Since $\mu_{\phi}(C)=\int \mathcal{L}_{0}\left(\mathbb{1}_{C}\right) d \mu_{\phi}$, it is enough to find a bound for $\mathcal{L}_{0}\left(\mathbb{1}_{C}\right)$. Since $\mathcal{L}_{0}(\mathbb{1})=\mathbb{1}$, it is easy to see that $\mathcal{L}_{0}\left(\mathbb{1}_{C}\right)$ can be bounded from above by $\prod_{j=1}^{k} \mathcal{L}_{0}^{\Delta m_{j}} \mathbb{1}_{W_{\Delta m_{j}}}$ The latter is estimated using the following.

Lemma 4.9 For every $m \in \mathbb{N}, \mathcal{L}_{0} \mathbb{1}_{W_{m}} \leq C_{q} \exp \left(-\operatorname{rqm} \frac{\beta}{2}\right)$.

Proof This follows from Corollary 2.5, applied for $n=r$ and from Corollary 2.6.

Now, since the total length of blocks, $\sum \Delta m_{i}$, is larger than $\frac{\alpha n}{2}$, this gives:

$$
\left.\sup \mathcal{L}_{0} \mathbb{1}_{C} \leq C_{q}^{n} \exp \left(-r q\left(\sum \Delta m_{i}\right) \frac{\beta}{2}\right)\right) \leq C_{q}^{n} \exp \left(-r q \alpha n \frac{\beta}{4}\right)
$$

Since there are at most $3^{n}$ possible configurations, this gives:

$$
\mu_{\phi}\left(Y_{n}\right) \leq\left(3 C_{q}\right)^{n} \exp (-r q n \beta \alpha / 4)
$$

Thus (4.6) holds if $r$ is large enough.

The proof of the next lemma is almost the same as the proof of Lemma 18 from [12]. The only modification is that $\partial U$ is to be replaced by $\partial B^{\prime}$.

Lemma 4.10 Let $R_{n} \subset B^{\prime}$ be the set of points in $x \in Z_{n}$ that satisfy the following two requirements.

(1) $x \in B \backslash Y_{n}$, i.e. the points in $R_{n}$ have at least $\frac{\alpha}{2} n$ good pullbacks, but

(2) No good pullback $V_{m}(x)$ with $m \leq n$, is very good.

Then

$$
\mu_{\phi}\left(R_{n}\right) \leq(3 C)^{n} \tilde{\lambda}^{-\alpha n / 2},
$$

where the constant $C$ (again: independent of $r$ ) comes from Lemma 4.11 below.

We omit the proof of this lemma, referring to [12], but, since the value of the constant $C$ in the statement of Lemma 4.10 is important, we explain it in Lemma 4.11.

Lemma 4.11 Denote by $G B_{k}^{h}$ the subfamily of good components for $h^{k}$, which are entirely contained in $B\left(\partial B^{\prime}, \lambda^{-k}\right)$. Then

$$
\sup \mathcal{L}_{0}^{k} \mathbb{1}_{G B_{k}} \leq C \tilde{\lambda}^{-k}
$$


where $C=\frac{C_{l}}{\mu_{\phi}\left(B^{\prime}\right)}$.

Proof We have

$$
\begin{aligned}
\tilde{\lambda}^{-k} \geq \mu_{\phi}\left(B\left(\partial B^{\prime}, \lambda^{-k}\right)\right) & =\int \mathbb{1}_{B\left(\partial B^{\prime}, \lambda^{-k}\right)} d \mu_{\phi} \geq \int \mathcal{L}_{0}^{k} \mathbb{1}_{G B_{k}} d \mu_{\phi} \\
& \geq \mu\left(B^{\prime}\right) \inf \mathcal{L}_{0}^{k} \mathbb{1}_{G B_{k}} \\
& \geq \frac{1}{C_{l}} \sup \mathcal{L}_{0}^{k} \mathbb{1}_{G B_{k}} \cdot \mu_{\phi}\left(B^{\prime}\right) .
\end{aligned}
$$

In order to make the measure $\mu_{\phi}\left(R_{n}\right)$ converge to zero exponentially fast, we thus require $\lambda$ to be so large that

$$
\lambda^{\frac{\alpha}{6}}=\tilde{\lambda}^{\frac{\alpha}{2}}>3 C
$$

(remember that $\lambda=\tilde{\lambda}^{3}$ ). Here, again, $C=\frac{C_{l}}{\mu_{\phi}\left(B^{\prime}\right)}$. So, denoting

$$
\lambda^{\prime \prime}=\tilde{\lambda}^{\frac{\alpha}{2}} \cdot \frac{1}{3 C}>1
$$

we have $\mu_{\phi}\left(R_{n}\right) \leq\left(\lambda^{\prime \prime}\right)^{-n}$.

The constants used in the construction. To clarify the situation, we summarize here the procedure of fixing consecutive constants (and iterates of the map $f$ ) used in the construction.

(1) The constant $l$ is taken from Lemma 2.2, applied for $\varepsilon=1 / 2$.

(2) The ball $B$ (and, consequently, also $B^{\prime}$ ) is chosen so that $B$ satisfies the assumptions of Lemma 2.2, and, moreover, so that the statement of Lemma 3.2 is satisfied, for every $\lambda$ large enough.

(3) The constant $q$ depends on $B$.

(4) The constant $\alpha$ depends on $q$.

(5) Since in the statement of Lemma $3.2 \lambda$ can be always replaced by its power, now, having fixed $q$ and $\alpha$, we fix $\lambda$ so large that (4.7) holds.

(6) Finally, $r$ is fixed. The iterate $r$ depends on $q, \alpha$ and $\lambda$.

\section{Construction of the induced system}

Let

$$
X=\bigcup_{n=1}^{\infty} Z_{n} \backslash\left(Y_{n} \cup R_{n}\right)
$$

It directly follows from Lemmas 4.2, 4.4, 4.8, 4.10 that

$$
\mu_{\phi}(X)=\mu_{\phi}\left(B^{\prime}\right) .
$$


Given $x \in X$ let $n(x)$ be the smallest integer $n \geq 1$ such that the pullback $V_{n}(x)$ is very good.

We define

$$
F(x)=h^{n(x)}(x) .
$$

Keep this $x \in X$ and put $n=n(x)$. Note that, if $y \in V_{n}(x)$ then this procedure, applied to $y$ leads to the same component $V_{n}$. Indeed, by the definition of the induced map, we use the earliest very good pullback. Thus, if $F(y) \neq h^{n}(y)$ then $F(y)=$ $h^{m}(y)$ for some $m<n$. Let $V_{m}(y)$ be the corresponding pullback. Then $V_{m}(y) \cap$ $V_{n}(x) \neq \varnothing$ as $y$ belongs to both of these sets, but $V_{n}(x) \nsubseteq V_{m}(y)$ since $x \in V_{n}(x) \backslash$ $V_{m}(y)$. Let us consider $h^{m}\left(V_{m}(y)\right)=U$ and $h^{m}\left(V_{n}(x)\right)$. The latter is an element of the pullback chosen for $x$, a component of $h^{-(n-m)}(B)$, and, since $V_{n}(x)$ must intersect $\partial V_{m}(y)$, also $h^{m}\left(V_{n}(x)\right)$ intersects $\partial B^{\prime}$. But this is impossible by the definition of very good pullbacks. Let $\mathcal{D}$ be the countable family of all sets $V_{n(x)}(x), x \in X$, defined in this way. We have just shown that the function $n: X \rightarrow \mathbb{N}$ is constant on each set $D \in \mathcal{D}$, and so it can and will be treated as a function from $\mathcal{D} \rightarrow \mathbb{N}$. In particular, the map

$$
F: \bigcup_{D \in \mathcal{D}} \rightarrow B^{\prime}
$$

is well-defined and its inverse branches $F_{D}^{-1}: B^{\prime} \rightarrow D, D \in \mathcal{D}$, form an infinite iterated function systems, which, with a slight abuse of notation, will be also referred to as $F$. We denote by $J_{F}$ the limit set of the iterated function system $F$, i.e.

$$
J_{F}=\bigcap_{n=0}^{\infty} F^{-n}(X) .
$$

The argument leading to (5.1) gives in fact more. Namely:

$$
\mu_{\phi}\left(J_{F}\right)=\mu_{\phi}\left(B^{\prime}\right)
$$

It immediately follows from the construction of the system $F$ and Lemma 4.10, that

$$
m_{\phi}(\bigcup\{D: n(D)=n\}) \leq\left(\lambda^{\prime \prime}\right)^{-n}
$$

for some $\lambda^{\prime \prime}>1$ and all $n \geq 1$. Let us record the following, proved in the same way as Lemma 19 in [12], essential property of this induced system.

Lemma 5.1 If $D_{1}, D_{2}$ are two domains in $\mathcal{D}, F_{\mid D_{1}}=h^{n}, F_{\mid D_{2}}=h^{m}$ then for $0 \leq$ $s<n, 0 \leq t<m$ either $h^{s}\left(D_{1}\right) \cap h^{t}\left(D_{2}\right)=\emptyset$ or the closure of one of these sets is contained in the other set.

For the sake of Proposition 5.4, we need to extend the potential $\phi$ beyond the Julia sets $J(f)$.

Lemma 5.2 The function $\phi$ can be extended in a Hölder continuous manner, with the same Hölder exponent, to the whole projective space $\mathbb{P}^{k}$. 
This lemma is well-known. From now on, we assume that the potential $\phi$ is defined and Hölder continuous in the whole projective space $\mathbb{P}^{k}$.

As we have passed to induced system, we shall modify the potential $\phi$ accordingly to this inducing process. First, if $D \in \mathcal{D}$ is one of the domains on which $F$ is defined, then we put, for all $x \in D$, that

$$
\hat{\phi}(x)=\sum_{k=0}^{n(D)-1} S_{q r} \phi\left(h^{k}(x)\right) .
$$

Then, for all Borel sets $A \subset D_{e}$ we have that,

$$
\begin{aligned}
m_{\phi}(F(A)) & =m_{\phi}\left(h^{n(D)}(A)\right)=\int_{A} \exp \left(-\sum_{k=0}^{n(D)-1} S_{q r} \phi \circ h^{k}\right) d m_{\phi} \\
& =\int_{A} \exp (-\hat{\phi}(x)) d m_{\phi}(x) .
\end{aligned}
$$

Along with (5.3) this entails the following.

Lemma 5.3 The probability measure $m_{\phi}$ is $\exp (-\hat{\phi})$-conformal for the map $F$ : $J_{F} \rightarrow J_{F}$.

Having Lemmas 5.3 and 5.2, the general theory of infinite iterated function systems, as developed in [8,9], see also [10], gives the following.

Proposition 5.4 There exists a unique probability $F$-invariant measure $\mu_{\hat{\phi}}$ which is equivalent to $m_{\phi}$. Moreover the Radon-Nikodym derivative $\hat{\rho}:=\frac{d \mu_{\hat{\phi}}}{d m_{\phi}}$ is bounded above and separated below from zero. This Radon-Nikodym derivative $\hat{\rho}$ has a continuous extension $\hat{\rho}: B^{\prime} \rightarrow(0 ;+\infty)$ to the whole ball $B^{\prime}$ and this extension is a fixed point of the following transfer operator.

$$
\mathcal{L}_{\hat{\phi}}(v)(x)=\sum_{y \in F^{-1}(x)} \exp \hat{\phi}(y) v(y) .
$$

This is a bounded linear operator acting on the Banach space $C_{b}(B)$ of all bounded real-valued continuous functions defined on $B$, and it is easy to see that this operator is almost periodic.

\section{Real analyticity of topological pressure}

For every Hölder continuous potential $\phi: J(f) \rightarrow \mathbb{R}$, let

$$
\Delta_{\phi}:=\left\{t \in \mathbb{R}: \sup (t \phi)-\inf (t \phi)<\kappa_{f}\right\},
$$


where $\kappa_{f}$ was defined in Theorem 1.1. Obviously, $\Delta_{\psi}$ is an open subset of $\mathbb{R}$. Having all the material of the previous sections i.e. Sects. 4 and 5, particularly formula (5.4), we can repeat verbatim Sect. 6, Real Analyticity of the Pressure Function, from [12] to get the following.

Theorem 6.1 The topological pressure function

$$
\Delta_{\phi} \ni t \mapsto P(t \phi) \in \mathbb{R}
$$

is real-analytic.

Remark 6.2 With only slight formal modification we could prove a little bit more; namely that for any Hölder continuous functions $\psi, \phi: J(f) \rightarrow \mathbb{R}$, the function

$$
\Delta_{\phi}(\psi) \ni t \mapsto P(\psi+t \phi) \in \mathbb{R}
$$

is real-analytic if $\Delta_{\phi}(\psi):=\left\{t \in \mathbb{R}: \sup (\psi+t \phi)-\inf (\psi+t \phi)<\kappa_{f}\right\}$.

\section{Stochastic properties of the equilibrium measure $\mu_{\phi}$}

In this section we obtain strong transparent stochastic properties of the dynamical system $\left(f, \mu_{\phi}\right)$. We deduce them from the corresponding properties of the induced system $\left(F, \mu_{\hat{\phi}}\right)$. We follow the scheme worked out in [13] in the way it was presented in [12]. We recall it briefly now. We do this in an abstract context. Let $\left(\Delta_{0}, \mathcal{B}_{0}, m_{0}\right)$ be a measure space with a finite measure $m_{0}$, let $\mathcal{P}_{0}$ be a countable measurable partition of $\Delta_{0}$ and let $T_{0}: \Delta_{0} \rightarrow \Delta_{0}$ be a measurable map such that, for every $\Delta^{\prime} \in \mathcal{P}_{0}$ the map $T_{0}: \Delta^{\prime} \rightarrow \Delta_{0}$ is a bijection onto $\Delta_{0}$. Moreover, we assume that the partition $\mathcal{P}_{0}$ is generating, i.e. for every $x, y \in \Delta_{0}$ there exists $s \geq 0$ such that $T_{0}^{s}(x), T_{0}^{s}(y)$ are in different elements of the partition $\mathcal{P}_{0}$. We denote by $s=s(x, y)$ the smallest integer with this property and we call it a separation time for the pair $x, y$. We assume also that for each $\Delta^{\prime} \in \mathcal{P}_{0}$ the map $\left(T_{0 \mid \Delta^{\prime}}\right)^{-1}$ is measurable and that the Jacobian $\operatorname{Jac}_{m_{0}}\left(T_{0}\right)$ with respect to the measure $m_{0}$ is well-defined and positive a.e. in $\Delta^{\prime}$. The following distortion property is assumed to be satisfied.

$$
\left|\frac{\mathrm{Jac}_{m_{0}} T_{0}(x)}{\mathrm{Jac}_{m_{0}} T_{0}(y)}-1\right| \leq C \kappa^{s(x, y)} .
$$

for all elements $\Delta^{\prime} \in \mathcal{P}_{0}$, all $x, y \in \Delta^{\prime}$ and some $\kappa \in(0,1)$. We have also a function $R: \Delta_{0} \rightarrow \mathbb{N}$ ("return time") which is constant on each element of the partition $\mathcal{P}_{0}$. We assume that the greatest common divisor of the values of $R$ is equal to 1. Finally, let

$$
\Delta=\left\{(z, n) \in \Delta_{0} \times \mathbb{N} \cup\{0\}: 0 \leq n<R(z)\right\}
$$

and each point $z \in \Delta_{0}$ is identified with $(z, 0)$. The map $T$ acts on $\Delta$ as

$$
T(z, n)=\left\{\begin{array}{lll}
(z, n+1) & \text { if } & n+1<R(z) \\
\left(T_{0}(z), 0\right) & \text { if } & n+1=R(z)
\end{array}\right.
$$


The measure $m_{0}$ is spread over the whole space $\Delta$ by putting

$$
\tilde{m}_{\mid \Delta_{0}}=m_{0} \quad \text { and } \quad \tilde{m}_{\mid \Delta^{\prime} \times\{j\}}=m_{0 \mid \Delta^{\prime}} \circ \pi_{j}^{-1}, \Delta^{\prime} \in \mathcal{P}_{0},
$$

where $\pi_{j}(z, 0)=(z, j)$. Thus, the measure $\tilde{m}$ is finite iff $\int_{\Delta_{0}} R d m_{0}<\infty$. The separation time $s((x, n),(y, m))$ is defined to be equal to $s(x, y)$ if $n=m$ and $x, y$ are in the same set of the partition $\mathcal{P}$. Otherwise we set $s(x, y)=0$. Given any $\beta \in(0,1)$ we define the space

$$
C_{\beta}(\Delta)=\left\{\varphi: \Delta \rightarrow \mathbb{R}: \exists C_{\varphi} \quad \text { suchthat }|\varphi(x)-\varphi(y)|<C_{\varphi} \beta^{s(x, y)} \quad \forall x, y \in \Delta\right\} .
$$

We refer to the pentapol $\mathcal{Y}=\left(\Delta_{0}, T_{0}, \mathcal{P}_{0}, R, m_{0}\right)$ as a Young tower. The first three items of the following basic result have been proved in [13], see also [7] for the fourth item.

Theorem 7.1 If $\mathcal{Y}=\left(\Delta_{0}, T_{0}, \mathcal{P}_{0}, R, m_{0}\right)$ is a Young tower and $\int R d m_{0}<\infty$ then the following hold.

(1) There exists a unique probability $T$-invariant measure $v$, absolutely continuous with respect to $\tilde{m}$. The Radon-Nikodym derivative $d v / d \tilde{m}$ is bounded from below by a positive constant. The dynamical system $(T, v)$ is exact, thus ergodic.

(2) If $m_{0}(R>n)=O\left(\theta^{n}\right)$ for some $0<\theta<1$, then for every $\beta>0$ there exists $0<\tilde{\theta}<1$, depending on $\beta$, such that for all functions $\psi \in L^{\infty}$ and all functions $g \in C_{\beta}$ we have,

$$
\operatorname{Cov}\left(\psi \circ T^{n}, g\right)=\left|\int\left(\psi \circ T^{n}\right) g d v-\int \psi d v \int g d v\right|=O\left(\tilde{\theta}^{n}\right)
$$

(3) If $m_{0}(R>n)=O\left(n^{-\alpha}\right)$ with some $\alpha>1$ (in particular, if $m_{0}(R>n)=O\left(\theta^{n}\right)$ ), then the Central Limit Theorem is satisfied for all functions $g \in C_{\beta}$, that are not cohomologous to a constant in $L^{2}(v)$.

(4) If $_{0}(R>n)=O\left(n^{-\alpha}\right)$ with some $\alpha>4\left(\right.$ in particular, if $\left.m_{0}(R>n)=O\left(\theta^{n}\right)\right)$, then the Law of Iterated Logarithm holds for all $\beta>0$ and all functions $g \in C_{\beta}$, that are not cohomologous to a constant in $L^{2}(v)$. This means that there exists a real positive number $A_{g}$ such that such that $v$ almost everywhere

$$
\limsup _{n \rightarrow \infty} \frac{S_{n} g-n \int g d v}{\sqrt{n \log \log n}}=A_{g}
$$

Passing to our holomorphic dynamical system $\left(f, \mu_{\phi}\right)$ we shall check that the assumptions of this theorem are satisfied for our induced system $\left(F, m_{\phi}\right)$. The space $\Delta_{0}$ is now $J_{F}$, the limit set of the iterated function system $F$. The partition $\mathcal{P}_{0}$ consists of the sets $D \cap J_{F}, D \in \mathcal{D}$. The measure $m_{0}$ is the conformal measure $m_{\phi}$, restricted to $J_{F}$. The map $T_{0}$ is, in our setting, the map $F$. The function $R$, the return time, is, naturally, defined as $R(D)=n(D)$. We shall check that the pentapol $\mathcal{Y}_{\phi}=\left(J_{F}, F, \mathcal{P}, n, m_{\phi}\right)$ is a Young Tower, i.e. it satisfies the hypotheses of Theorem 7.1. We start with the following. 
Lemma 7.2 There exists a constant $C>0$ such that if $D \in \mathcal{D}$ and $x, y \in D$, then

$$
\operatorname{dist}(x, y) \leq C 4^{-s(x, y)}
$$

Proof The assertion follows immediately from Corollary 2.3, formula (4.1), and the definition of the separation time $s$.

As a fairly straightforward consequence of this lemma, we get the following.

Lemma 7.3 There exists a constant $C^{\prime}>0$ such that if $D \in \mathcal{D}$ and $x, y \in D$, then

$$
\operatorname{dist}\left(h^{j}(x), h^{j}(y)\right) \leq C^{\prime}(1 / 4)^{n(D)-j+s(x, y)}
$$

for all $0 \leq j \leq n(D)-1$.

Proof Recall that for each $D \in \mathcal{D}$ we have $\left.F\right|_{D}=h^{n(D)}$ and $h$ expands by a factor equal at least 4 on each set $h^{i}(D)$ for all $i=0, \ldots, n-1$. Therefore we can very generously estimate,

$$
\operatorname{dist}\left(h^{j}(x), h^{j}(y) \leq(1 / 4)^{n(D)-j} \operatorname{dist}(F(x), F(y))\right.
$$

By virtue of Lemma 7.2, this yields

$$
\operatorname{dist}\left(h^{j}(x), h^{j}(y) \leq(1 / 4)^{n(D)-j} C 4^{-s(F(x), F(y))} .\right.
$$

Now, if $s(x, y)>1$, then $s(F(x), F(y))=s(x, y)-1$, and if $s(x, y)=1$, then $s(F(x), F(y)) \geq s(x, y)-1$. In any case inserting this to the last display, gives

$$
\operatorname{dist}\left(h^{j}(x), h^{j}(y) \leq 4 C(1 / 4)^{n(D)-j+s(x, y)} .\right.
$$

The proof is complete.

Lemma 7.4 The pentapol $\mathcal{Y}_{\phi}=\left(J_{F}, F, \mathcal{P}, n, m_{\phi}\right)$ is a Young Tower, i.e. it satisfies the hypotheses of Theorem 7.1. In addition, $\tilde{m}_{\phi}(\Delta)<+\infty$.

Proof First, we need to show that the formula (7.1) holds. To do this fix an arbitrary domain $D \in \mathcal{D}$ and arbitrary two points $x, y \in J_{F} \cap D$. Recalling that the function $S_{q r} \phi: J(f) \rightarrow \mathbb{R}$ is Hölder continuous with some exponent $\alpha>0$, and using Lemma 7.3, we can write as follows. 


$$
\begin{aligned}
\left|\log \frac{\mathrm{Jac}_{m_{\phi}} F(y)}{\mathrm{Jac}_{m_{\phi}} F(x)}\right| & =\left|\sum_{j=0}^{n(D)-1} S_{q r} \phi\left(h^{j}(y)\right)-\sum_{j=0}^{n(D)-1} S_{q r} \phi\left(h^{j}(x)\right)\right| \\
& \leq \sum_{j=0}^{n(D)-1}\left|S_{q r} \phi\left(h^{j}(y)\right)-S_{q r} \phi\left(h^{j}(x)\right)\right| \\
& \leq \sum_{j=0}^{n(D)-1} C_{1} \operatorname{dist}^{\alpha}\left(h^{j}(y), h^{j}(x)\right) \\
& \leq C_{1} C^{\prime} \sum_{j=0}^{n(D)-1}(1 / 4)^{n(D)-j+s(x, y)} \\
& =C_{1} C^{\prime}(1 / 4)^{s(x, y)} \sum_{j=0}^{n(D)-1}(1 / 4)^{n(D)-j} \\
& \leq C_{1} C^{\prime}(1 / 4)^{s(x, y)} \sum_{j=0}^{\infty}(1 / 4)^{j} \\
& =(4 / 3) C_{1} C^{\prime}(1 / 4)^{s(x, y)}
\end{aligned}
$$

and formula (7.1) is established.

We also need to take care of the last assumption in Theorem 7.1 requiring that the greatest common divisor of all the values of $n(D), D \in \mathcal{D}$, is equal to 1 . If for our induced system this value is equal to some integer $s>1$, then we replace the map $h$ by its iterate $h^{s}$. The return times are now equal to $n(D) / s, D \in \mathcal{D}$, and their greatest common divisor equals 1 .

The finiteness of $\tilde{m}_{\phi}(\Delta)$ follows immediately from (5.4) and the definition of the return time $R$.

Now consider $\pi: \Delta \rightarrow \mathbb{P}^{k}$, the natural projection from the abstract tower $\Delta$ to the projective space $\mathbb{P}^{k}$ given by the formula

$$
\pi(z, n)=h^{n}(z)
$$

Then

$$
\begin{aligned}
& \pi \circ T=h \circ \pi, \\
& \left.\tilde{m_{\phi}}\right|_{J_{F}} \circ \pi^{-1}=m_{0}=m_{\phi},
\end{aligned}
$$

and

$$
\tilde{m}_{\phi \mid D \times\{n\}} \circ \pi^{-1}=m_{\phi \mid D \times\{0\}} \circ h^{-n}=m_{0 \mid D} \circ h^{-n}
$$

for all $D \in \mathcal{D}$ and all $0 \leq n \leq n(D)$. Now, the measure $\tilde{m}_{\phi \mid D \times\{n\}} \circ \pi^{-1}$ is absolutely continuous with respect to $m_{\phi}$ with the Radon-Nikodym derivative equal to $J_{D, n}:=\operatorname{Jac}_{m_{\phi}}\left(h^{-n}\right)$ in $h^{n}(D)$ and zero elsewhere. Therefore, 


$$
\begin{aligned}
\int_{\mathbb{P}^{k}} \sum_{D \in \mathcal{D}} \sum_{n=0}^{n(D)-1} J_{D, n} d m_{\phi} & =\sum_{D \in \mathcal{D}} \sum_{n=0}^{n(D)-1} \int_{\mathbb{P}^{k}} J_{D, n} d m_{\phi}=\sum_{D \in \mathcal{D}} \sum_{n=0}^{n(D)-1} \int_{h^{n}(D)} J_{D, n} d m_{\phi} \\
& =\sum_{D \in \mathcal{D}} \sum_{n=0}^{n(D)-1} \tilde{m}_{\phi \mid D \times\{n\}} \circ \pi^{-1}\left(h^{n}(D)\right) \\
& =\sum_{D \in \mathcal{D}} \sum_{n=0}^{n(D)-1} \tilde{m}_{\phi \mid D \times\{n\}} \circ \pi^{-1}\left(\mathrm{P}^{k}\right) \\
& =\tilde{m}_{\phi} \circ \pi^{-1}\left(\mathbb{P}^{k}\right)=\tilde{m}_{\phi}(\Delta) \\
& <+\infty,
\end{aligned}
$$

where in writing the inequality sign we used the last assertion of Lemma 7.4. Thus, the function $\sum_{D \in \mathcal{D}} \sum_{0 \leq n<n(D)} J_{D, n}$ is integrable with respect to the measure $m_{\phi}$. This implies immediately that the measure $\tilde{m}_{\phi} \circ \pi^{-1}$ is absolutely continuous with respect to the measure $m_{\phi}$ with the Radon-Nikodym derivative equal to $\sum_{D \in \mathcal{D}} \sum_{0 \leq n<n(D)} J_{D_{i}, n}$. Hence, the measure $\nu \circ \pi^{-1}$ is also absolutely continuous with respect to $m_{\phi}$. Since $v$ is $F$-invariant and $\pi \circ T=h \circ \pi$, the measure $v \circ \pi^{-1}$ is $h$-invariant. But the measure $\mu_{\phi}$ is $h$-invariant ergodic and equivalent with the conformal measure $m_{\phi}$. Hence, $v \circ \pi^{-1}$ is absolutely continuous with respect to the ergodic measure $\mu_{\phi}$. Invariance and ergodicity of $\nu \circ \pi^{-1}$ yield this.

\section{Lemma 7.5}

$$
v \circ \pi^{-1}=\mu_{\phi}
$$

Having this, we can prove in exactly the same way as in [12], the following.

Theorem 7.6 For the dynamical system $\left(f, \mu_{\phi}\right)$ the following hold.

(1) For every $\alpha \leq 1$, every $\alpha$-Hölder continuous function $g: J(f) \rightarrow \mathbb{R}$ and every bounded measurable function $\psi: J(f) \rightarrow \mathbb{R}$, we have that

$$
\left|\int \psi \circ f^{n} \cdot g d \mu_{\phi}-\int g d \mu_{\phi} \int \psi d \mu_{\phi}\right|=O\left(\theta^{n}\right)
$$

for some $0<\theta<1$ depending on $\alpha$.

(2) The Central Limit Theorem holds for every Hölder continuous function $g$ : $J(f) \rightarrow \mathbb{R}$ that is not cohomologous to a constant in $L^{2}\left(\mu_{\phi}\right)$, i.e. for which there is no square integrable function $\eta$ for which $g=$ const $+\eta \circ f-\eta$. Precisely this means that there exists $\sigma>0$ such that

$$
\frac{1}{\sqrt{n}} \sum_{j=0}^{n-1} g \circ f^{j} \rightarrow \mathcal{N}(0, \sigma)
$$

in distribution. 
(3) The Law of Iterated Logarithm holds for every Hölder continuous function $g$ : $J(f) \rightarrow \mathbb{R}$ that is not cohomologous to a constant in $L^{2}\left(\mu_{\phi}\right)$. This means that there exists a real positive constant $A_{g}$ such that such that $\mu_{\phi}$ almost everywhere

$$
\limsup _{n \rightarrow \infty} \frac{S_{n} g-n \int g d \mu}{\sqrt{n \log \log n}}=A_{g} .
$$

Open Access This article is distributed under the terms of the Creative Commons Attribution License which permits any use, distribution, and reproduction in any medium, provided the original author(s) and the source are credited.

\section{References}

1. Briend, J.-Y., Duval, J.: Deux caractérisations de la mesure d'équilibre d'un endomorphisme de $P^{k}(\mathbb{C})$. Publ. Math. Inst. Hautes Etudes Sci. 93, 145-159 (2001)

2. Denker, M., Urbański, M.: Ergodic theory of equilibrium states for rational maps. Nonlinearity 4, 103-134 (1991)

3. Dinh, T., Sibony, N.: Dynamics in several complex variables: endomorphisms of projective spaces and polynomial-like mapping. Lecture Notes in Mathematics 1998 (2010)

4. Dupont, Ch.: Bernoulli coding map and almost sure invariance principle for endomorphisms of $\mathbb{C P}^{k}$. Probab. Theor. Relat. Fields 196, 337-359 (2010)

5. Dupont, Ch.: Large entropy measures for endomorphisms of $\mathbb{C P}^{k}$. Isr. J. Math. 192, 505-533 (2012)

6. Guedj, V.: Ergodic properties of rational mappings with large topological degree. Ann. Math. (2) 161, 1589-1607 (2005)

7. Melbourne, I., Nicol, M.: Almost sure invariance principle for nonuniformly hyperbolic systems. Commun. Math. Phys. 260, 131-146 (2005)

8. Mauldin, D., Urbański, M.: Dimensions and measures in infinite iterated function systems. Proc. Lond. Math. Soc. (3) 73, 105-154 (1996)

9. Mauldin, D., Urbański, M.: Graph Directed Markov Systems: Geometry and Dynamics of Limit Sets. Cambridge University Press, Cambridge (2003)

10. Mauldin, D., Urbański, M.: On the uniqueness of the density of the invariant measure in an infinite hyperbolic iterated function system. Periodica Math. Hung. 37, 47-53 (1998)

11. Przytycki, F., Urbański, M.: Conformal Fractals: Ergodic Theory Methods. Cambridge University Press, Cambridge (2010)

12. Szostakiewicz, M., Urbański, M., Zdunik, A.: Fine Inducing and Equilibrium Measures for Rational Functions of the Riemann Sphere (preprint). http://www.math.unt.edu/ urbanski

13. Young, L.S.: Recurrence times and rates of mixing. Isr. J. Math. 110, 153-188 (1999)

14. Sibony, N., Wong, P.M.: Some results on global analytic sets, in Séminaire Pierre Lelong-Henri Skoda (Analyse). Années 1978/79, Lecture Notes in Math., vol. 822, pp. 221-237

15. Urbański, M., Zdunik, A.: Equilibrium measures for holomorphic endomorphisms of complex projective space. Fund. Math. 220, 23-69 (2013) 